\title{
ARTICLE
}

Neurological Classification of SCI

\section{Classification challenges of the 2019 revised International Standards for Neurological Classification of Spinal Cord Injury (ISNCSCI)}

\author{
Steven Kirshblum ${ }^{1,2} \cdot$ Mary Schmidt $\operatorname{Read}^{3} \cdot$ Rüdiger Rupp $\mathbb{D}^{4}$ \\ Received: 24 January 2021 / Revised: 18 May 2021 / Accepted: 19 May 2021 / Published online: 4 June 2021 \\ (c) The Author(s) 2021. This article is published with open access
}

\begin{abstract}
Study design Retrospective review of ISNCSCI datasets.

Objectives To discuss the correct classification of ISNCSCI datasets considered as challenging.

Setting International expert collaboration.

Methods The International Standards Committee of the American Spinal Injury Association (ASIA) receives challenging case scenarios regarding the International Standards for the Neurological Classification of Spinal Cord Injury (ISNCSCI). Among those cases received, sample cases representing different categories of typical classification difficulties were identified by members of the International Standards committee.

Results From the cases received, five sample cases were identified as representative for publication. These cases are related to the correct classification in the presence of non-SCI related conditions, the determination of motor zones of partial preservation in regions with no myotomes to test, the classification of the ASIA Impairment Scale in patients with substantial motor function below the motor level but no sacral sparing, the inclusion of non-key muscle functions in the classification of sensory incomplete individuals, and the correct classification of individuals with an amputation.

Conclusion Presenting cases with challenging classifications, along with responses and explanations, will serve spinal cord injury professionals to better understand and utilize the ISNCSCI classification. As the ISNCSCI endorsed by ASIA and the International Spinal Cord Society (ISCoS) evolves over time, such resources are important to clarify inquiries from the spinal cord injury community and to understand the rationale for revisions.
\end{abstract}

\section{Introduction}

Since the introduction of the original Standards for the Classification of Spinal Cord Injuries by the American Spinal Injury Association (ASIA) in 1982 [1], the International Standards for the Neurological Classification of Spinal Cord Injury (ISNCSCI) has undergone a number of

\footnotetext{
Supplementary information The online version contains supplementary material available at https://doi.org/10.1038/s41393021-00648-y.

Rüdiger Rupp

ruediger.rupp@med.uni-heidelberg.de

Kessler Institute for Rehabilitation, West Orange, NJ, USA

2 Rutgers New Jersey Medical School, Newark, NJ, USA

3 Magee Rehabilitation/Jefferson Health, Philadelphia, PA, USA

4 Spinal Cord Injury Center, Heidelberg University Hospital, Heidelberg, Germany
}

revisions [2-5], including most recently in 2019 as fully described in other articles in this issue. The ISNCSCI endorsed by ASIA and the International Spinal Cord Society (ISCoS) is the most widely used classification in the field of spinal cord injury (SCI) medicine, and describes the examination and definitions to be used for clinical and research purposes around the world.

The ASIA International Standards Committee often receives inquiries regarding the ISNCSCI. In January of 2019, a call for challenging cases to be submitted to the committee was communicated via e-mail to all ASIA membership. These questions are usually handled by the Chair of the committee along with other committee members to assure a consensus response, and then sent directly to the person(s) who posed the question. Previous cases, as well as other challenging questions regarding the ISNCSCI, have been published to serve as a resource for other SCI professionals to consult $[6,7]$.

In this paper, we describe five case scenarios that represent challenging questions with their explanations; the 
explanations include some of the Standard revisions introduced in 2019. The questions include (1) how to score a complicated case when a tagged score would make the difference between an ASIA Impairment Scale (AIS) C and $\mathrm{D}$; (2) how to score the motor zone of partial preservation (ZPP) in the thoracic area with varied sensory scores; (3) can the sacral sparing definition be used to classify the injury as complete even if the individual can ambulate; (4) how to classify a case with non-key muscles documented; and (5) how to classify a case with an amputation above the suspected level of injury.

\section{Methods}

Fifteen cases were submitted to the committee as a response to the call. These cases were related to problems with the classification of two spinal injuries, concomitant non-SCI conditions, amputations, not determinable AIS/Neurological Level of Injury (NLI) due to non-testable (NT) being documented in decisive segments, autonomous reactions on deep anal pressure (DAP), ambulatory AIS A patients, and other general challenging cases. Among those cases received by the International Standards Committee and responded to, sample cases representing different categories of typical classification difficulties were identified and included here. For each of the cases received, some committee members (including the authors) reviewed the questions. One member would draft a response that was evaluated by other review members. Any differences of opinion would be discussed until consensus was obtained. In all cases, there was unanimous agreement of the response.

\section{Results}

\section{Challenging case 1}

With adoption of the 2019 revisions to the International Standards, what is the best way to classify a case when there is a myotome not grading as normal due to an old non-SCIrelated injury (old tibia-fibula fracture with peripheral nerve damage), with the motor score of that specific myotome impacting the determination of the AIS (Fig. 1)? In this case, does the AIS need to be recorded as "ND"?

\section{Response}

From a clinical perspective, the left L5 myotome can be considered as "normal for classification purposes". This is documented on the worksheet by adding an asterisk $(*)$ to the left L5 motor score and providing the reason for the asterisk $(*)$ and how to handle it for classification as additional information in the comments box. As such, even

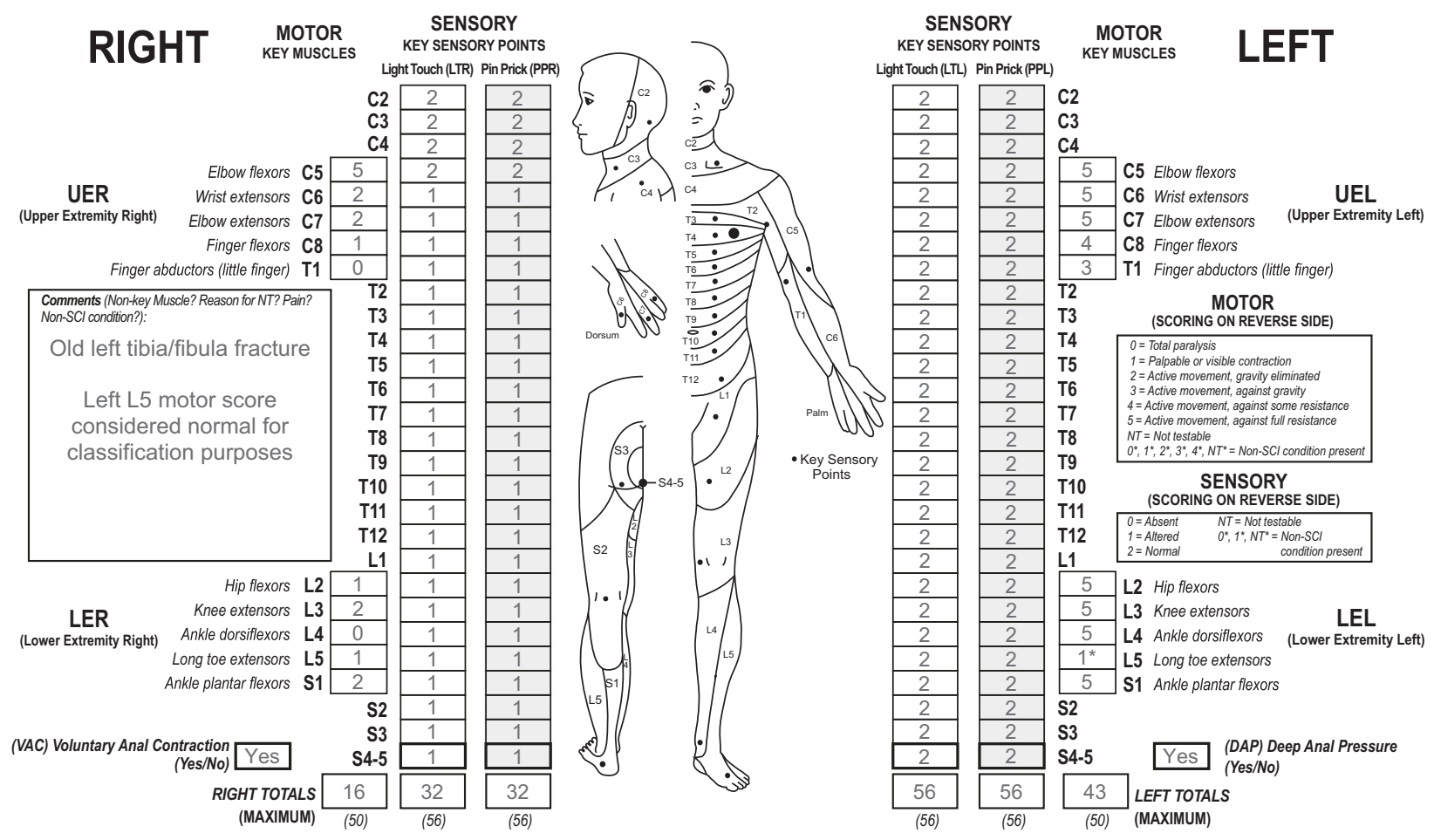

Fig. 1 Case 1 with a non-SCI condition superimposed to the SCI. This individual has an unimpaired sensory function on the left side and a motor impairment of the left long toe extensor due to an old tibia

fracture documented as $1 *$ in the left L5 segment. Based on the clinical assumption, this asterisk (*)-tagged score should be considered as normal during classification, which is noted in the comments box. 


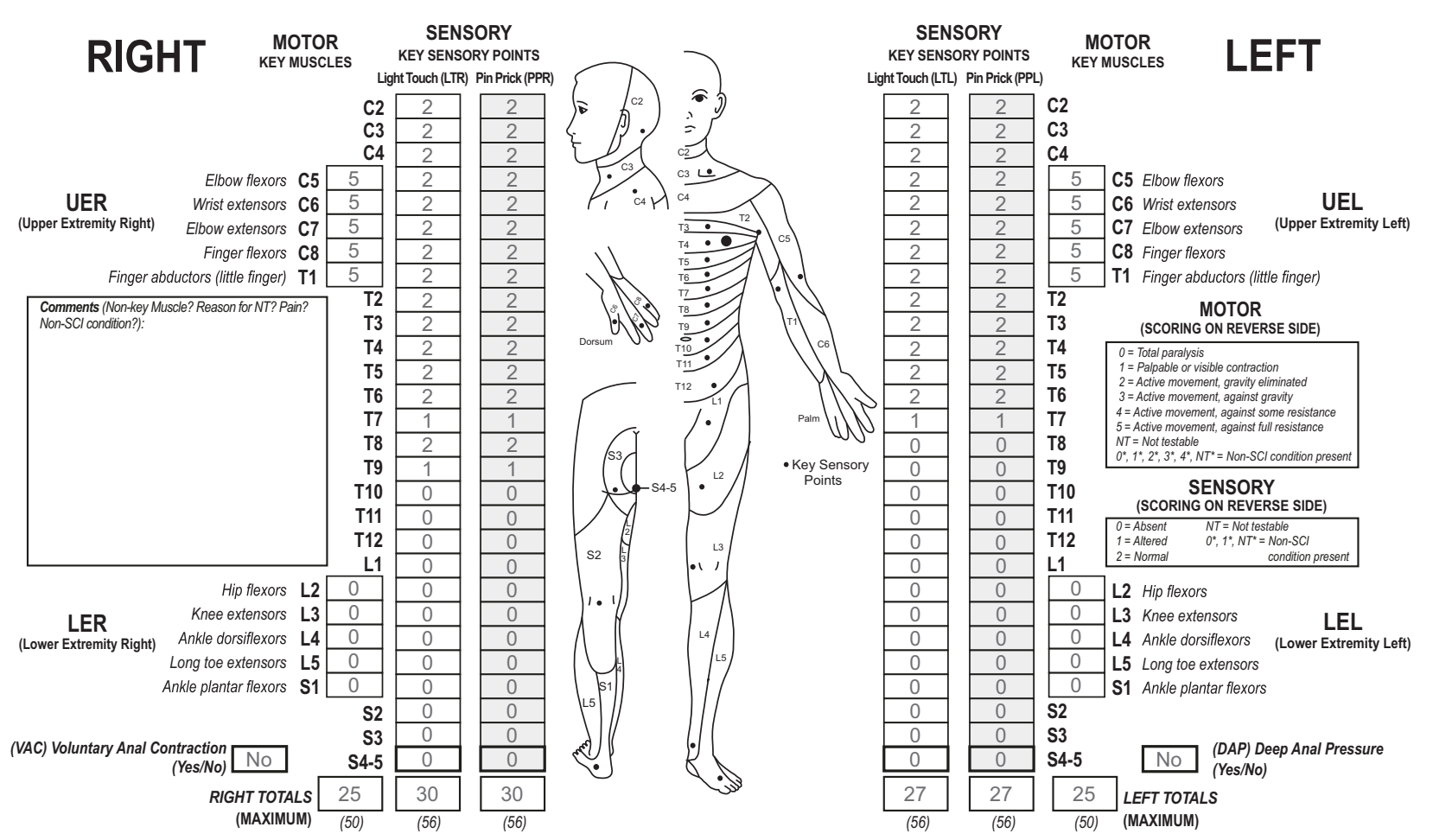

Fig. 2 Case 2 with a complete SCI and preserved functions below the lesion level. This individual has a complete (AIS A) thoracic lesion with some sensory functions preserved below the NLI.

though there is an abnormal motor score $(1 *)$ in left L5, this muscle is considered as a "normal" grade $(=5)$ for classification. Since this individual example has a NLI at C5, a motor score of $\geq 3 / 5$ in $\geq 9$ of the remaining myotomes below the NLI (18 of them) would lead to an AIS classification of AIS D, and less than 9 would lead to an AIS classification of AIS C. In this case scenario, there are nine key muscles below the NLI that have a strength of at least $3 / 5$, including this L5 myotome. As such, the classification would be designated as an AIS D. Importantly, since this classification was made based upon a clinical assumption (the tagged score for the left L5 key muscle group), the designation of AIS D should be tagged (AIS D*). While for classification purposes the '*'-tagged motor score is replaced by a normal score this is not true for calculating the sum motor score of the left side. The sum scores are always based on the examined score. This results in a total motor score on the left side of 43 .

A challenge in this case could occur if the examiner documented in the comment box that the $1^{*}$ for the left L5 myotome-is "not considered as normal". While this determines that the score is not considered a 5 , the $1 *$ could represent a score of any grade from 1 to 4 . In this case, the grade of the myotome would make a difference in AIS classification. Specifically, if the $1^{*}$ would be considered a grade of a 1 or 2 , then the classification would be an AIS C (since less than $50 \%$ of the key muscles below the NLI would be considered to have a strength of $\geq 3 / 5$ ), and if a 3 or 4 , then an AIS D, as described above. Since either of these two scenarios are possible if marked as "not considered normal", the appropriate designation for the AIS would be documented as "ND*" (not determinable), with the tagged grade signifying that this grade is based upon a tagged score in a key muscle.

\section{Challenging case 2}

What is the right motor ZPP in a case where the sensory level is in the thoracic region, and sensation is intact at a level below the sensory level as seen in the example in the worksheet (Fig. 2)? As the rule is that the motor level defers to the sensory level in the thoracic area, would the right motor ZPP be $\mathrm{T} 8$ or T6?

\section{Response}

In the case presented (Fig. 2), the sensory level is T6, and the motor level (as well as the NLI) is also T6. The motor level defers to the sensory level since all key muscle functions at $\mathrm{T} 1$ and above are all intact. The sensory ZPP on the right is $\mathrm{T} 9$, however, the motor $\mathrm{ZPP}$ on the right remains at T6. Even though there is intact sensation at T8 on the right, one does not infer that motor function at $\mathrm{T} 8$ is also normal. In 2011, the rule for motor ZPP was clarified that "motor function does NOT follow sensory function in recording ZPP" [8]. This remained unchanged in the 2019 


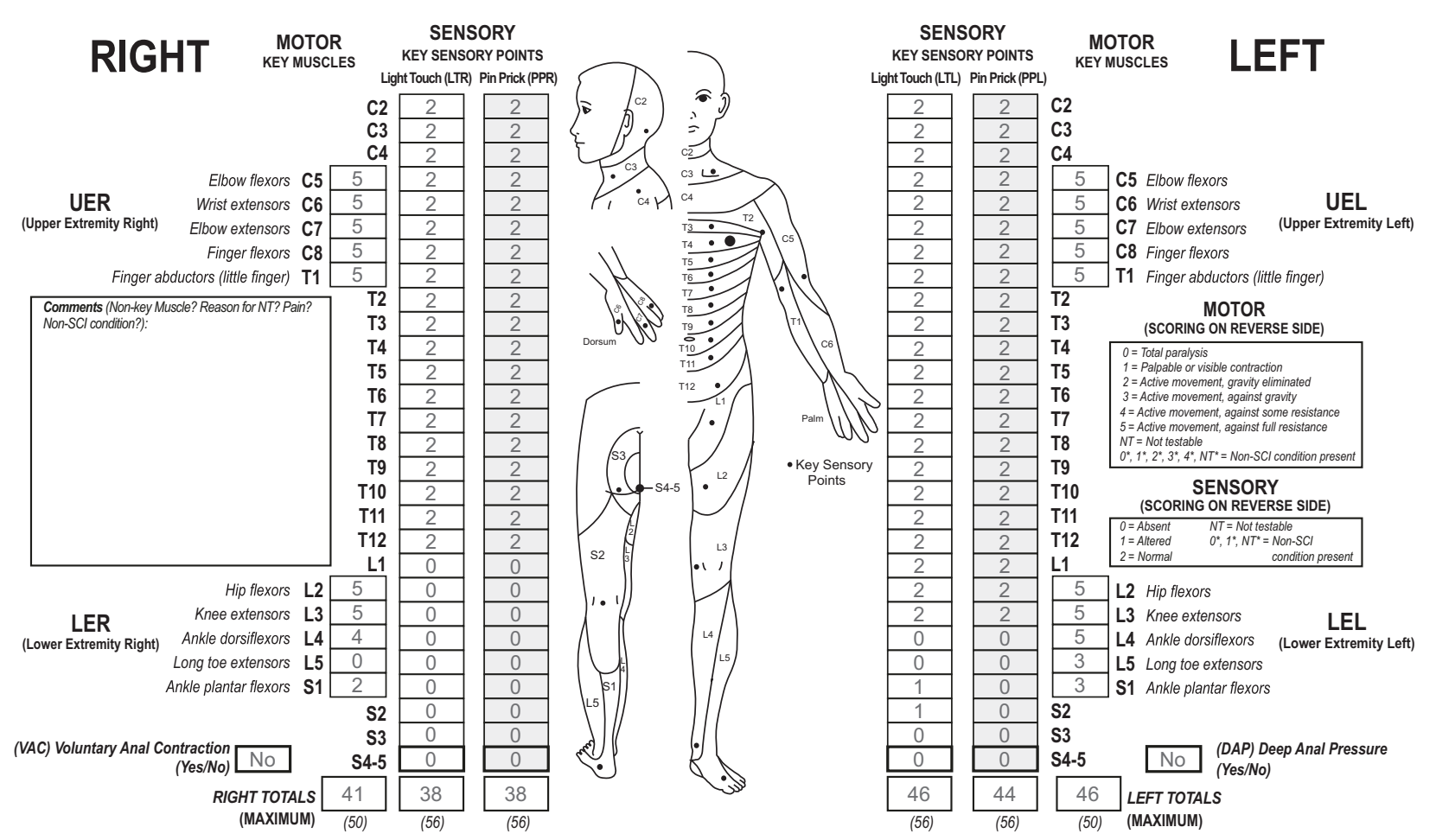

Fig. 3 Case 3 of an ambulatory person with a complete SCI. This individual has substantial motor functions preserved below the motor level, but no sacral sparing of sensory or motor functions.

revision [4]. The "caudal extent of the motor ZPP must be based on the presence of voluntary muscle contraction below the motor level". While the motor level defers to the sensory level in the regions where there is no key muscle function to test (C1-C4, T2-L1, S2-S4/5), motor ZPP does not defer to the sensory ZPP. In this case, the defined motor level is T6, with no apparent voluntary muscle action in key muscles below that T6 right motor level, therefore the right motor ZPP is T6.

\section{Challenging case 3}

How would you classify a case with a neurological level of T12 where there is a significant amount of motor sparing more than three levels below the motor level on each side but the individual does not have sacral sparing of sensory and motor functions (see Fig. 3)? Is the sacral sparing definition still used and this individual classified as complete (AIS A) even if he or she is able to walk?

\section{Response}

Since there is no sacral sparing present in this case scenario, including light touch and pin sensation at S4-5, DAP, or voluntary anal contraction (VAC), this individual would be classified as a neurologically complete injury (AIS A). While it may seem counterintuitive that a person with a neurological complete injury can ambulate, the purpose of ISNCSCI is the determination of the neurological level and severity of a SCI and is not intended to be a functional measure. The constellation of "AIS D-like" capabilities in terms of walking with a classification of AIS A therefore can occur, although these cases are uncommon. One report found that this occurred in $3.2 \%$ of the overall AIS A population within the first year after injury [9]. For this specific case based upon the level of injury, one could consider that this may represent a cauda equina injury with some lower motor neuron damage findings especially of the anal sphincter, although this designation does not impact whether the injury is classified as neurologically complete or incomplete. Because of missing sensory and motor function in the lowest sacral segments, sensory (right T12, left S2) and motor ZPPs (right S1, left S1) are given and allow for characterization of the extent of preserved functions below the NLI of T12.

\section{Challenging case 4}

How to classify an individual with a T2 sensory and motor level, with sparing of light touch and pin prick sensation on the left side only at the S4-5 dermatome and DAP, with no key muscle functions spared in bilateral lower extremities nor VAC, but the presence of voluntary right adductor muscle strength is noted (see Fig. 4)? 


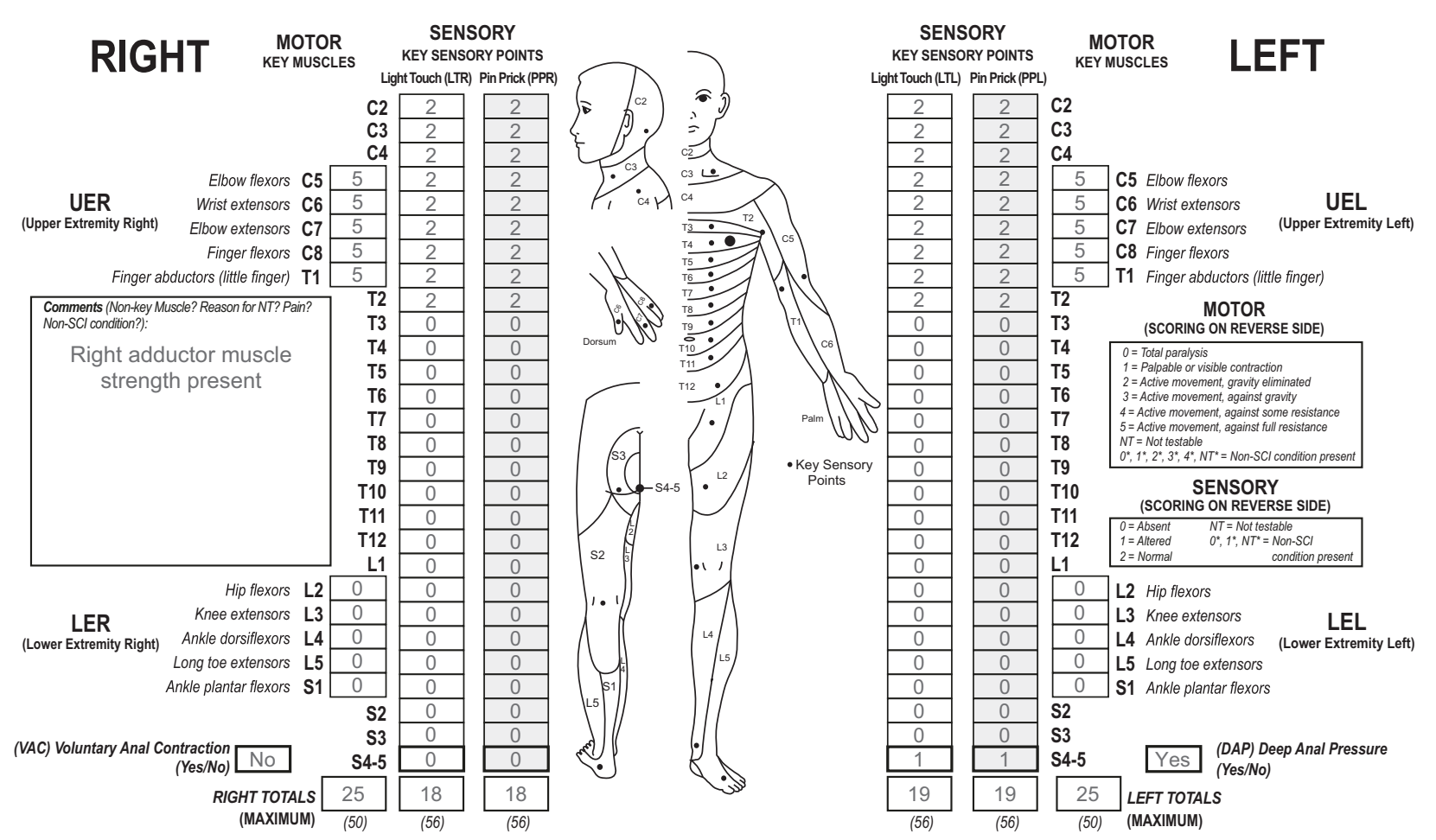

Fig. 4 Case 4 with preserved sensory function in lowest sacral segments and present motor function in a non-key muscle below the lesion level. This individual has a high-thoracic lesion with spared

sensory function in S4-5 on the left side and motor function in a nonkey muscle (hip adductor) on the right side.

\section{Response}

The motor and sensory level as well as the NLI are T2. The presence of DAP, and left LT and PP sensation at S4-5, allows for classification as at least an AIS grade B. While there is no VAC present, the presence of the right adductor muscle, whose innervation (L2) is more than three segments below the motor level $\mathrm{T} 2$, allows this case scenario of a sensory incomplete SCI to meet the criteria for classification as an AIS grade $\mathrm{C}$.

In this case, while sensory ZPPs on both sides are not applicable because of the presence of DAP, motor ZPPs are defined due to the missing VAC. Because preserved nonkey muscle function led to an AIS C classification, L2 as the most distal spinal segment with preserved motor function is recorded as the motor ZPP (L2) on the right side. It should be emphasized that the most distal non-key muscle function is only recorded as the motor ZPP in those exceptional situations when the non-key muscle function(s) are used to determine an AIS C classification. On the left side, T2 is recorded as the motor ZPP.

\section{Challenging case 5}

In a case scenario where a patient's right upper extremity underwent a transradial amputation below the level of the

elbow, the key muscles and key sensory points distal to the elbow cannot be tested and would be graded as NT. How would you classify an individual with this amputation, who otherwise presents with what appears to be a T6 level (see Fig. 5)?

\section{Response}

The motor and sensory scores of those spinal segments affected of the right upper extremity secondary to the forearm amputation should be documented as $\mathrm{NT}^{*}$. The reason for the tagged scores is due to the impairment (the amputation) not being SCI related. In the comments box, the reason for the NTs and the tagged ('*') scores are documented, as well as whether the scores for the myotomes and dermatomes should be documented as normal for classification. Since the remaining aspects of the neurological picture reveal a T6 level of injury, the clinical decision is made that these myotomes and dermatomes would grade normal for classification. As such, the right motor and sensory level is graded as $\mathrm{T} 6^{*}$, to follow the rule that levels changed because of tagged scores, should receive a tag. While the left motor and sensory levels would be documented as T6, and not require a tag, the NLI is documented as $\mathrm{T} 6^{*}$, because the right motor and sensory levels of $\mathrm{T}^{*}$ are based on clinical assumptions and so does the single NLI. 


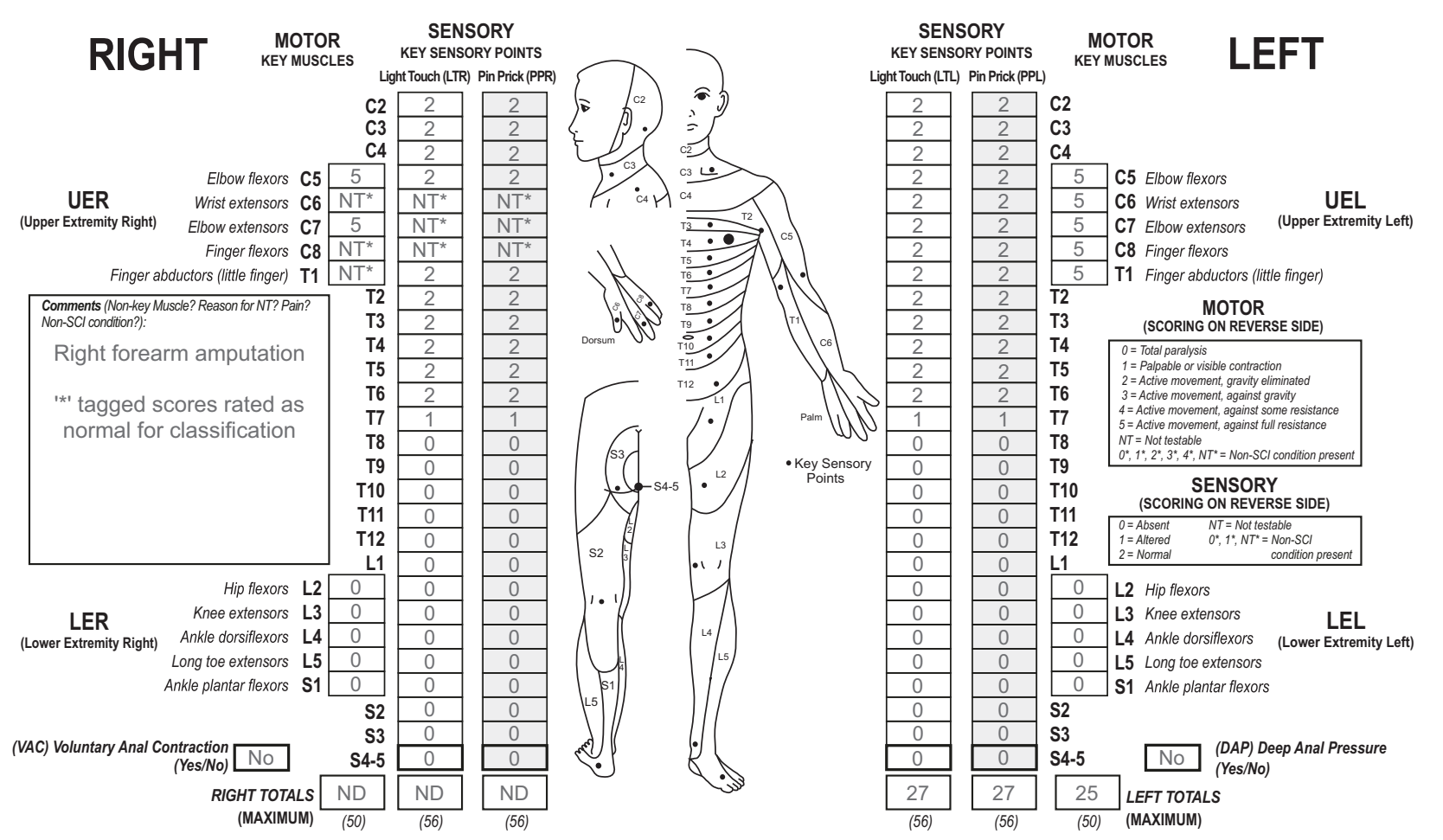

Fig. 5 Case 5 with a non-SCI condition above the lesion level. This individual has a mid-thoracic lesion and a transradial amputation of the right upper extremity. Based on the clinical assumption, these

The complete classification results of the five challenging cases can be found in the Supplementary Figs. 1-5.

\section{Discussion}

These challenging cases offer reinforcement to a number of important concepts in classifying SCI. This includes some of the most recent revisions in the ISNCSCI in 2019 utilizing tagged scores, reinforcing complicated concepts with the ZPP, reinforcing the rules of the sacral sparing definition of a complete injury, how to use non-key muscles in classifying incomplete injuries, and how to classify an individual with an amputation above the SCI lesion.

Over the years, the ISNCSCI has undergone revisions and updates that are aimed to improve the classification based upon feedback from professionals in the SCI community. The reinforcement of some older recommendations, as well as the more recent introduction of the non-SCI taxonomy, we believe facilitates better documentation of challenging cases that are found clinically. Review of InSTep (https://asia-spinalinjury.org/instep/) and continued training is of utmost importance for consistent usage of these Standards. The ASIA International Standards Committee invites the SCI community to share more challenging asterisk (*)-tagged NT scores on the right arm should be considered as normal during classification, which is noted in the comments box.

cases to continuously improve the ISNCSCI and the documentation of the neurological findings.

\section{Conclusion}

Presenting cases with challenging classifications, along with responses and explanations, will assist professionals serving the SCI population to better understand and utilize the ISNCSCI classification system. As the ISNCSCI evolves over time, such resources are important to clarify inquiries from the SCI community and to understand the rationale for revisions.

Acknowledgements We would like to thank all members of the AISA International Standards Committee for the support and discussions.

Author contributions SK compiled the first draft of the manuscript. All authors contributed to its content and were involved in the review and final approval process.

Funding Open Access funding enabled and organized by Projekt DEAL.

\section{Compliance with ethical standards}

Conflict of interest The authors declare no competing interests. 
Publisher's note Springer Nature remains neutral with regard to jurisdictional claims in published maps and institutional affiliations.

Open Access This article is licensed under a Creative Commons Attribution 4.0 International License, which permits use, sharing, adaptation, distribution and reproduction in any medium or format, as long as you give appropriate credit to the original author(s) and the source, provide a link to the Creative Commons license, and indicate if changes were made. The images or other third party material in this article are included in the article's Creative Commons license, unless indicated otherwise in a credit line to the material. If material is not included in the article's Creative Commons license and your intended use is not permitted by statutory regulation or exceeds the permitted use, you will need to obtain permission directly from the copyright holder. To view a copy of this license, visit http://creativecommons.org/licenses/by/4.0/.

\section{References}

1. American Spinal Injury Association. Standards for neurological classification of spinal injury patients. Chicago, IL: ASIA; 1982.

2. Kirshblum S, Waring W. Updates for the International Standards for Neurological Classification of Spinal Cord Injury. Phys Med Rehabil Clin N. 2014;25:505-17.
3. Rupp R, Biering-Sørensen F, Burns SP, Graves DE, Guest J, Jones $\mathrm{L}$, et al. International Standards for Neurological Classification of Spinal Cord Injury: Revised 2019. Top Spinal Cord Inj Rehabil. 2021;27:1-22. https://doi.org/10.46292/sci2702-1.

4. American Spinal Injury Association. International standards for neurological classification of spinal cord injury. Richmond: ASIA: 2019.

5. ASIA and ISCoS International Standards Committee. The 2019 revision of the International Standards for Neurological Classification of Spinal Cord Injury (ISNCSCI) - What's new? Spinal Cord. 2019. https://doi.org/10.1038/s41393-019-0350-9.

6. Kirshblum SC, Biering-Sorensen F, Betz R, Burns S, Donovan W, Graves DE, et al. International Standards for Neurological Classification of Spinal Cord Injury: cases with classification challenges. J Spinal Cord Med. 2014;37:120-7.

7. Solinsky R, Kirshblum SC. Challenging questions regarding the International Standards. J Spi Cord Med. 2018;41:684-90.

8. Kirshblum SC, Waring W, Biering-Sorensen F, Burns SP, Johansen M, Schmidt-Read M, et al. Reference for the 2011 revision of the International Standards for Neurological Classification of Spinal Cord Injury. J Spinal Cord Med. 2011;34:547-54.

9. Zariffa J, Curt A, EMSCI Study Group, Steeves JD. Functional motor preservation below the level of injury in subjects with American Spinal Injury Association Impairment Scale grade A spinal cord injuries. Arch Phys Med Rehabil. 2012;93:905-7. 